\title{
Tri-iodide Reduction Activity of Shape- and \\ Composition-Controlled PtFe Nanostructures as Counter \\ Electrodes in Dye-Sensitized Solar Cells
}

Pei-Jen Chang, ${ }^{1,2, \dagger}$ Kum-Yi Cheng, ${ }^{1,2, \dagger}$ Shang-Wei Chou, ${ }^{*}, 1$ Jing-Jong Shyue, ${ }^{3}$ Ya-Yun Yang, ${ }^{4}$ Chang-Yu Hung, ${ }^{1}$ Ching-Yen Lin, ${ }^{4}$ Hui-Lung Chen, ${ }^{5}$ Hung-Lung Chou, ${ }^{*} 6$ and Pi-Tai Chou ${ }^{*}, 1,2$

1. Department of Chemistry, National Taiwan University, Taipei, Taiwan, 10617;

2. Center for Emerging Material and Advanced Devices, National Taiwan University, Taipei, 10617 Taiwan

3. Department of Materials Science and Engineering, National Taiwan University, Taipei, Taiwan, 10617

4. Instrumentation Center, National Taiwan University, Taipei, 10617 Taiwan

5. Department of Chemistry and Institute of Applied Chemistry, Chinese Culture University, Taipei, 111 Taiwan

6. Graduate Institute of Applied Science \& Technology, National Taiwan University of Science and Technology, Taipei, 106, Taiwan

$\dagger$ Authors have equal contribution.

Corresponding author email:

sweichou47@ntu.edu.tw;

HLCHOU@mail.ntust.edu.tw;

chop@@ntu.edu.tw 


\section{Table of Content}

Supplementary Figures:

Figure S1: EDS and size distribution of $\mathrm{Pt}_{9} \mathrm{Fe}_{1}$ nanostructures.................................3

Figure S2: EDS and size distribution of $\mathrm{Pt}_{7} \mathrm{Fe}_{3}$ nanostructures..............................4

Figure S3: EDX Elemental analysis of $\mathrm{Pt}_{9} \mathrm{Fe}_{1}$ and $\mathrm{Pt}_{7} \mathrm{Fe}_{3}$ polyhedrons ..................5

Figure S4: EDX Elemental analysis of $\mathrm{Pt}_{9} \mathrm{Fe}_{1}$ and $\mathrm{Pt}_{7} \mathrm{Fe}_{3}$ nanocubes ......................6

Figure S5: EDX Elemental analysis of $\mathrm{Pt}_{9} \mathrm{Fe}_{1}$ and $\mathrm{Pt}_{7} \mathrm{Fe}_{3}$ concave cubes ................ 7

Figure S6: HRTEM images of $\{111\}$ and $\{100\}$ plane of $\mathrm{Pt}_{9} \mathrm{Fe}_{1}$ polyhedron ...........8

Figure S7: HRTEM images of $\{111\}$ and $\{100\}$ plane of $\mathrm{Pt}_{7} \mathrm{Fe}_{3}$ polyhedron ..........9

Figure S8: HRTEM images of $\mathrm{Pt}_{9} \mathrm{Fe}_{1}$ and $\mathrm{Pt}_{7} \mathrm{Fe}_{3}$ concave cube.............................10

Figure S9: Tafel curves of three-zone diagram ...........................................................11

Figure S10: Photovoltaic parameters of DSSC device using $\mathrm{Pt}_{9} \mathrm{Fe}_{1}$ nanostructures

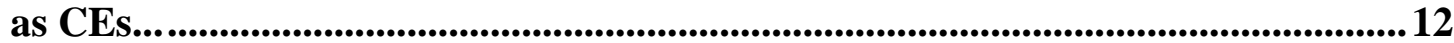

Figure S11: Photovoltaic parameters of DSSC device using $\mathrm{Pt}_{7} \mathrm{Fe}_{3}$ nanostructures as CEs ..............................................................................................................................13

Figure S12: $\mathrm{CV}$ of $\mathrm{Pt}_{9} \mathrm{Fe}_{1}$ and $\mathrm{Pt}_{7} \mathrm{Fe}_{3}$ nanostructures in $\mathrm{O.1}^{\mathrm{M} \mathrm{HClO}} \mathrm{H}_{4} \ldots \ldots . . . . . . . . . . . .14$

Figure S13: Theoretical model of $\mathrm{Pt}(111), \mathrm{Pt}_{9} \mathrm{Fe}_{1}(111)$, and (c) $\mathrm{Pt}_{9} \mathrm{Fe}_{1}(100) \ldots . . . .15$

\section{Supplementary Tables:}

Table S1. The synthetic parameters of PtFe nanostructures................................16

Table S2. Summary of $\mathbf{H}_{2}$-desorption area, electric charge, active area, and specific area of different counter electrodes

Table S3. Summary of adsorption energies of $\mathbf{I}_{2}$, desorption energies of $\mathrm{I}^{-}$, corresponding absolute energies and optimized geometries calculation 18

Table S4. Bader Charger Analysis 

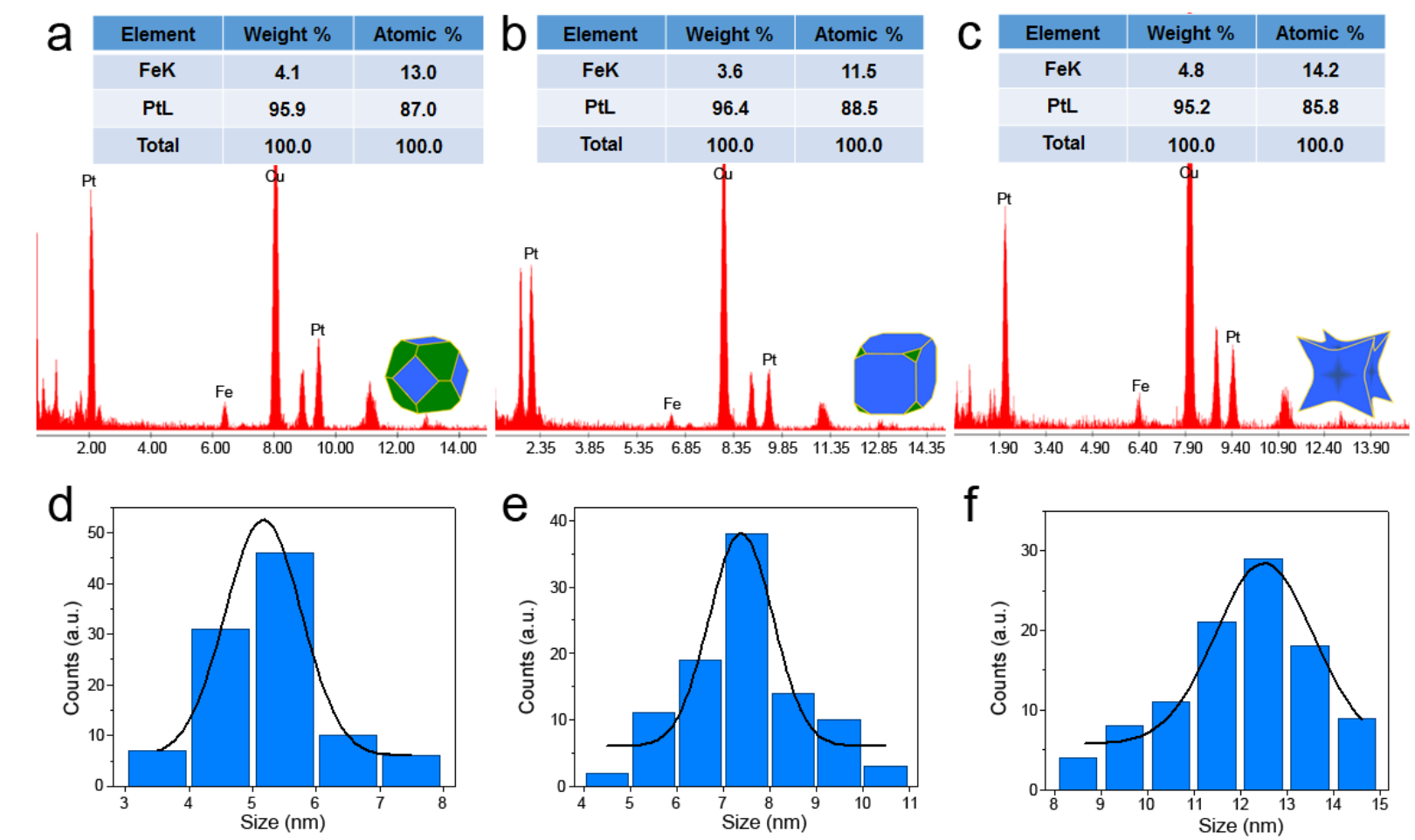

Figure S1. X-ray energy-dispersive spectra (EDS) of (a) $\mathrm{Pt}_{9} \mathrm{Fe}_{1}$ polyhedron, (b) $\mathrm{Pt}_{9} \mathrm{Fe}_{1}$ nanocube, and (c) $\mathrm{Pt}_{9} \mathrm{Fe}_{1}$ concave cube. Further, the distribution of the size of (d) $\mathrm{Pt}_{9} \mathrm{Fe}_{1}$ polyhedron, (e) $\mathrm{Pt}_{9} \mathrm{Fe}_{1}$ nanocube, and (f) $\mathrm{Pt}_{9} \mathrm{Fe}_{1}$ concave cube. 

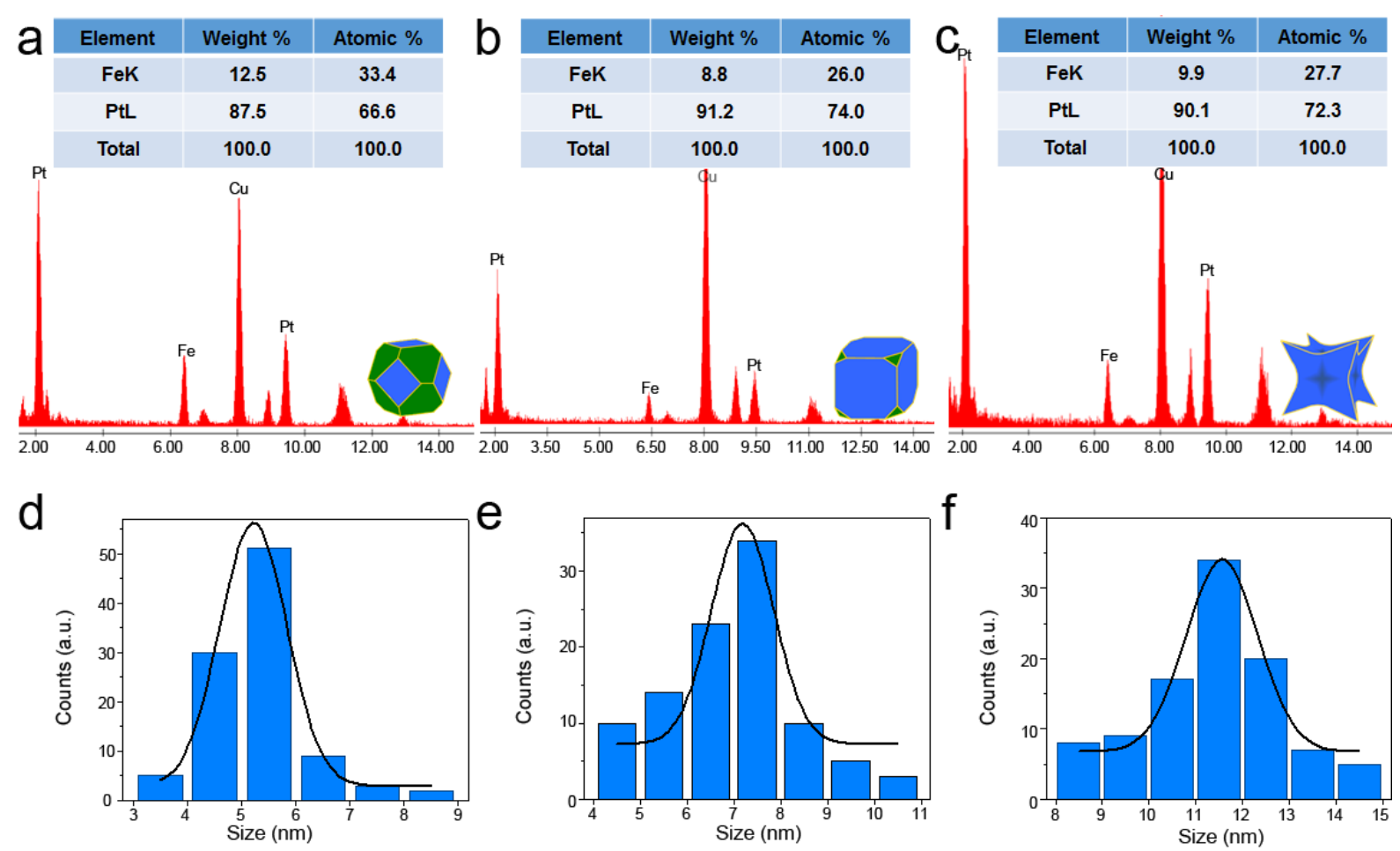

Figure S2. X-ray energy-dispersive spectra (EDS) of (a) $\mathrm{Pt}_{7} \mathrm{Fe}_{3}$ polyhedron, (b) $\mathrm{Pt}_{7} \mathrm{Fe}_{3}$ nanocube, and (c) $\mathrm{Pt}_{7} \mathrm{Fe}_{3}$ concave cube. Further, the distribution of the size of (d) $\mathrm{Pt}_{7} \mathrm{Fe}_{3}$ polyhedron, (e) $\mathrm{Pt}_{7} \mathrm{Fe}_{3}$ nanocube, and (f) $\mathrm{Pt}_{7} \mathrm{Fe}_{3}$ concave cube. 


\section{(a)}
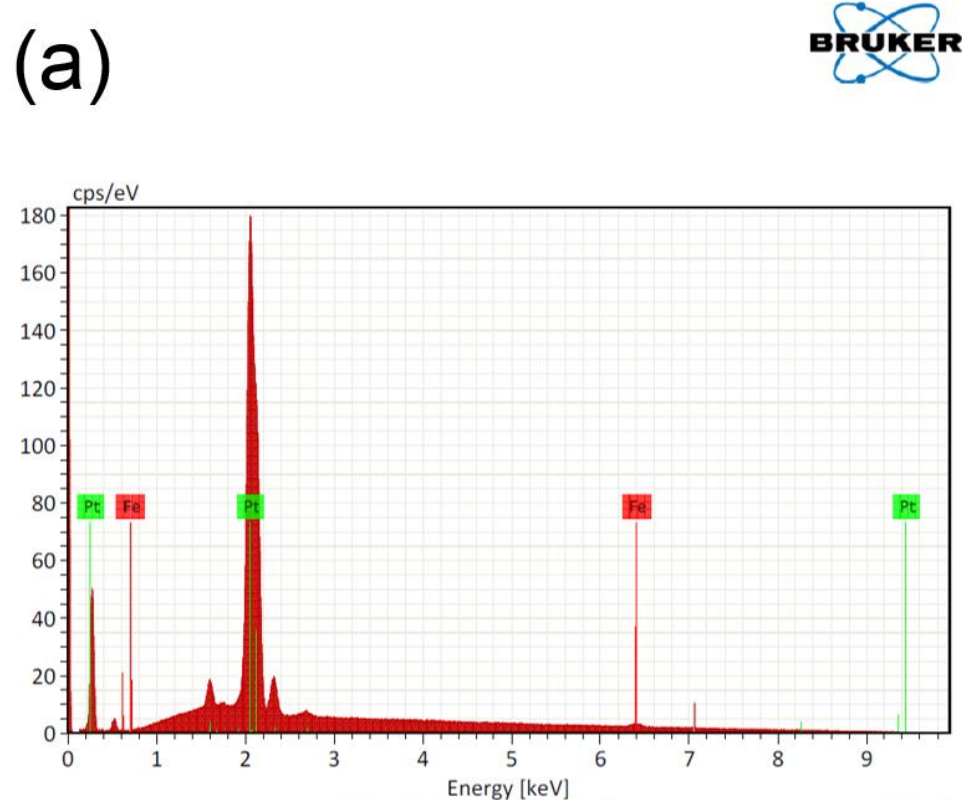

\begin{tabular}{|c|c|c|c|c|c|c|c|c|}
\hline Element & At. No. & Line s. & Netto & $\begin{array}{c}\text { Mass Norm. } \\
{[\%]}\end{array}$ & $\begin{array}{l}\text { Atom } \\
{[\%]}\end{array}$ & $\begin{array}{l}\text { abs. error [\%] } \\
\text { (1 sigma) }\end{array}$ & $\begin{array}{l}\text { abs. error [\%] } \\
\text { (2 sigma) }\end{array}$ & $\begin{array}{l}\text { abs. error [\%] } \\
\text { (3 sigma) }\end{array}$ \\
\hline Iron & 26 & K-Serie & 4839 & 4.48 & 14.08 & 0.21 & 0.41 & 0.62 \\
\hline Platinum & 78 & M-Serie & 658628 & 95.52 & 85.92 & 3.07 & 6.13 & 9.20 \\
\hline
\end{tabular}

\section{(b) BRUूer}

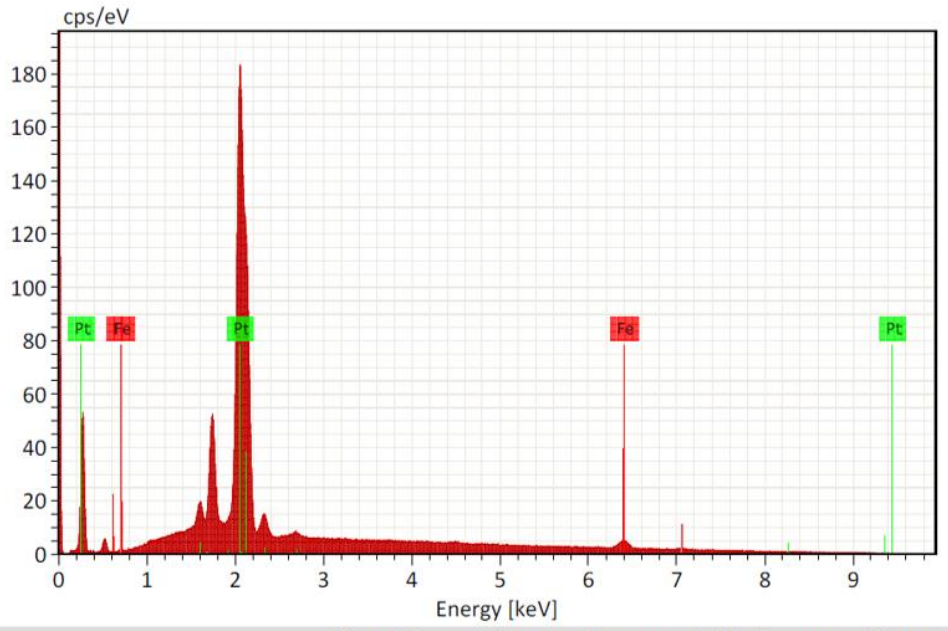

\begin{tabular}{|c|c|c|c|c|c|c|c|c|}
\hline Element & At. No. & Line s. & Netto & $\begin{array}{c}\text { Mass Norm. } \\
{[\%]}\end{array}$ & $\begin{array}{c}\text { Atom } \\
{[\%]}\end{array}$ & $\begin{array}{c}\text { abs. error [\%] } \\
\text { (1 sigma) }\end{array}$ & $\begin{array}{c}\text { abs. error [\%] } \\
\text { (2 sigma) }\end{array}$ & $\begin{array}{c}\text { abs. error [\%] } \\
\text { (3 sigma) }\end{array}$ \\
\hline Iron & 26 & K-Serie & 12972 & 11.97 & 32.20 & 0.45 & 0.90 & 1.35 \\
\hline Platinum & 78 & M-Serie & 656161 & 88.03 & 67.80 & 2.65 & 5.29 & 7.94 \\
\hline & & & Sum & 100.00 & 100.00 & & & \\
\hline
\end{tabular}

Figure S3. The EDX elemental analyses of (a) $\mathrm{Pt}_{9} \mathrm{Fe}_{1}$ polyhedron and (b) $\mathrm{Pt}_{7} \mathrm{Fe}_{3}$ polyhedron.

Note: the EDS are performed with an X-ray energy-dispersive spectrometer (model: QUANTAX Annular XFlash® QUAD FQ5060). 


\section{(a)}
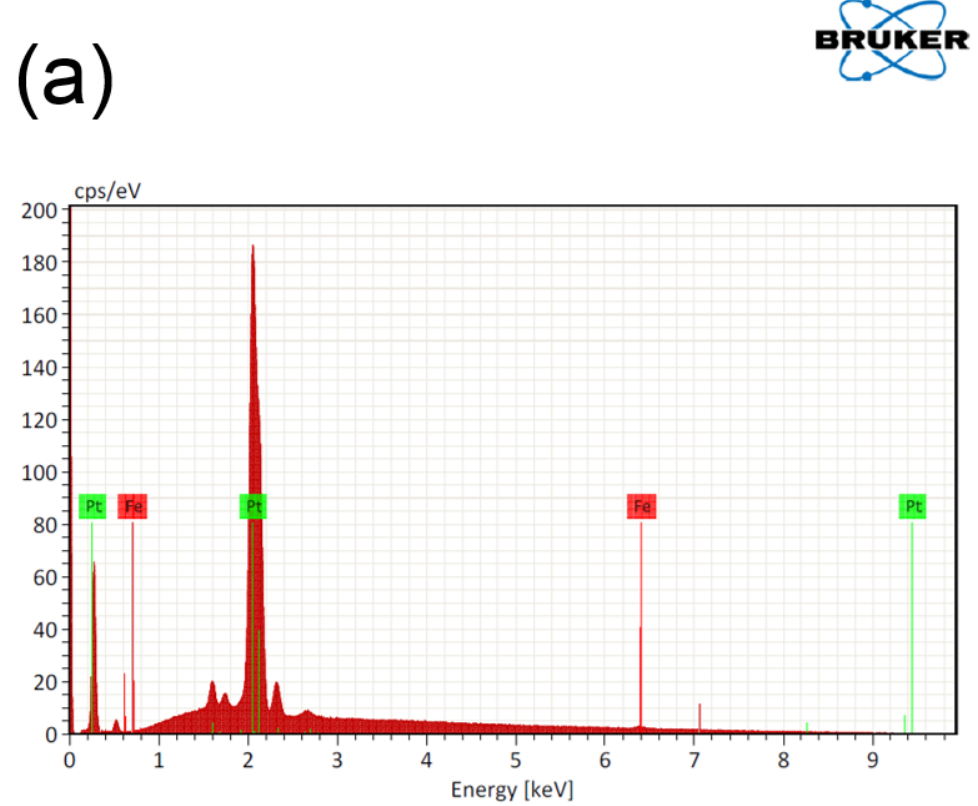

\begin{tabular}{|c|c|c|c|c|c|c|c|c|}
\hline Element & At. No. & Line s. & Netto & $\begin{array}{c}\text { Mass Norm. } \\
{[\%]}\end{array}$ & $\begin{array}{c}\text { Atom } \\
{[\%]}\end{array}$ & $\begin{array}{c}\text { abs. error [\%] } \\
\text { (1 sigma) }\end{array}$ & $\begin{array}{c}\text { abs. error [\%] } \\
\text { (2 sigma) }\end{array}$ & $\begin{array}{c}\text { abs. error [\%] } \\
\text { (3 sigma) }\end{array}$ \\
\hline on & 26 & K-Serie & 3336 & 3.54 & 11.37 & 0.16 & 0.32 & 0.48 \\
\hline atinum & 78 & M-Serie & 689555 & 96.46 & 88.63 & 2.83 & 5.67 & 8.50 \\
\hline
\end{tabular}

\section{(b) BRUER}

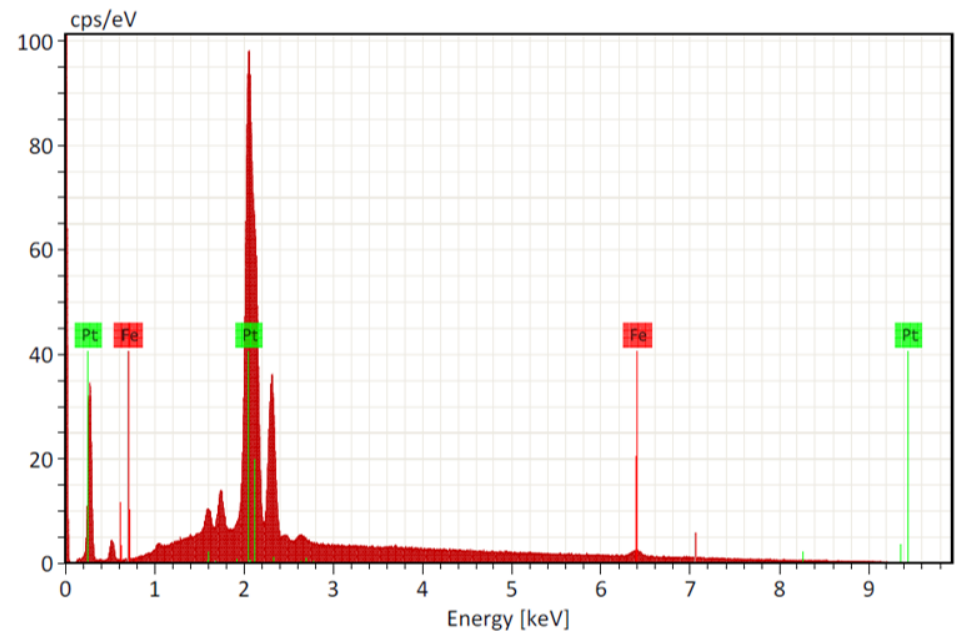

Elems Norm. Atom abs. error [\%] abs. error [\%] abs. error [\%]

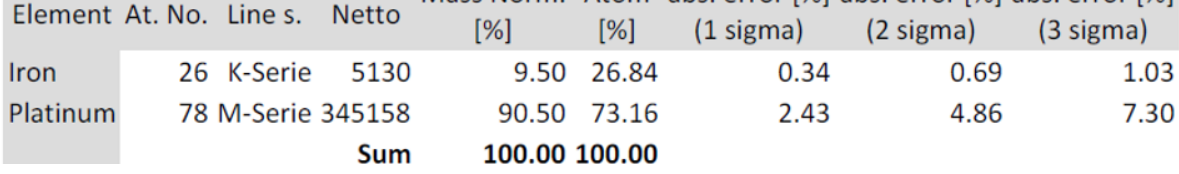

Figure S4. The EDX elemental analyses of (a) $\mathrm{Pt}_{9} \mathrm{Fe}_{1}$ nanocube and (b) $\mathrm{Pt}_{7} \mathrm{Fe}_{3}$ nanocube.

Note: the EDS are performed with an X-ray energy-dispersive spectrometer (model: QUANTAX Annular XFlash® QUAD FQ5060). 


\section{(a)}

\section{BRUKER}

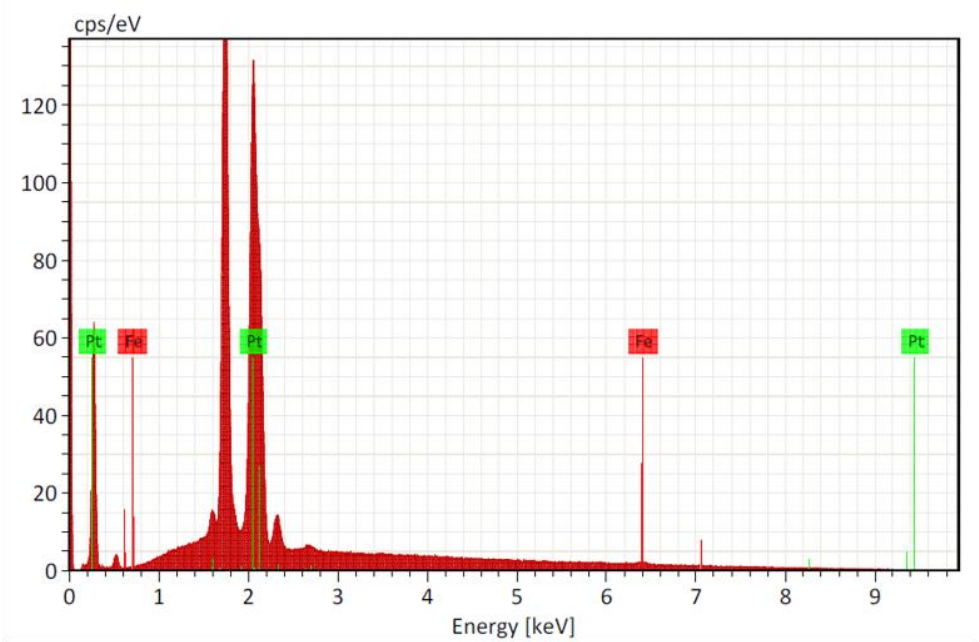

Element At. No. Line s. Netto Mass Norm. Atom abs. error [\%] abs. error [\%] abs. error [\%]

\begin{tabular}{|c|c|c|c|c|c|c|c|c|}
\hline Element & No. & Line s. & Netto & [\%] & [\%] & (1 sigma) & (2 sigma) & (3 sigma) \\
\hline Iron & 26 & K-Serie & 2637 & 4.88 & 15.23 & 0.17 & 0.34 & 0.51 \\
\hline Platinum & 78 & M-Serie & 411949 & 95.12 & 84.77 & 2.08 & 4.15 & 6.23 \\
\hline & & & Sum & 100.00 & 100.00 & & & \\
\hline
\end{tabular}

(b)

BRUKER

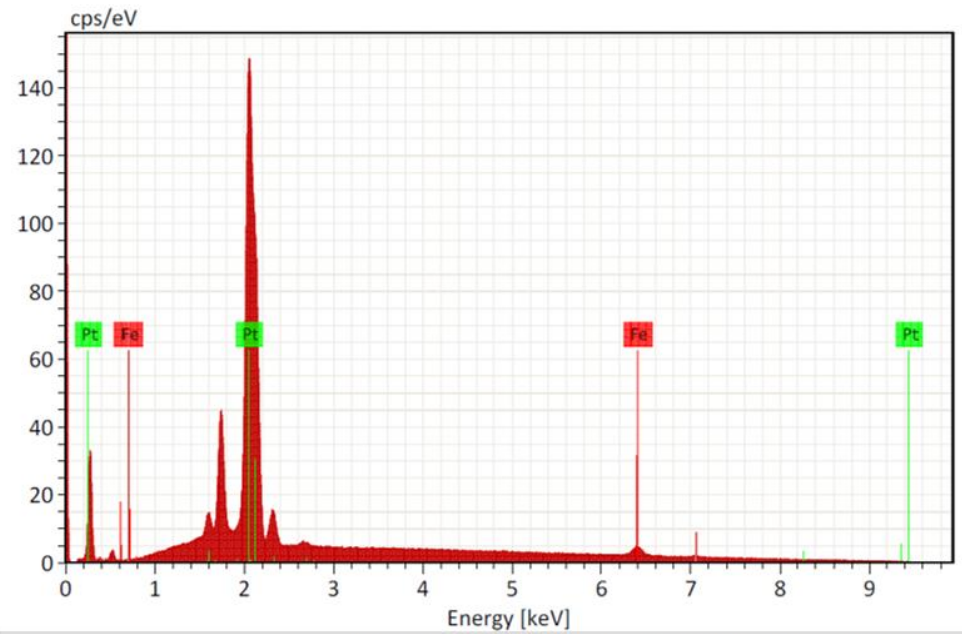

Element At No. Line s. Netto Mass Norm. Atom abs. error [\%] abs. error [\%] abs. error [\%]

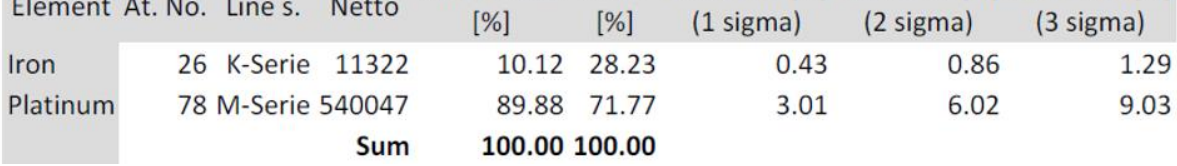

Figure S5. The EDX elemental analyses of (a) $\mathrm{Pt}_{9} \mathrm{Fe}_{1}$ concave cube and (b) $\mathrm{Pt}_{7} \mathrm{Fe}_{3}$ concave cube.

Note: the EDS are performed with an X-ray energy-dispersive spectrometer (model: QUANTAX Annular XFlash® QUAD FQ5060). 

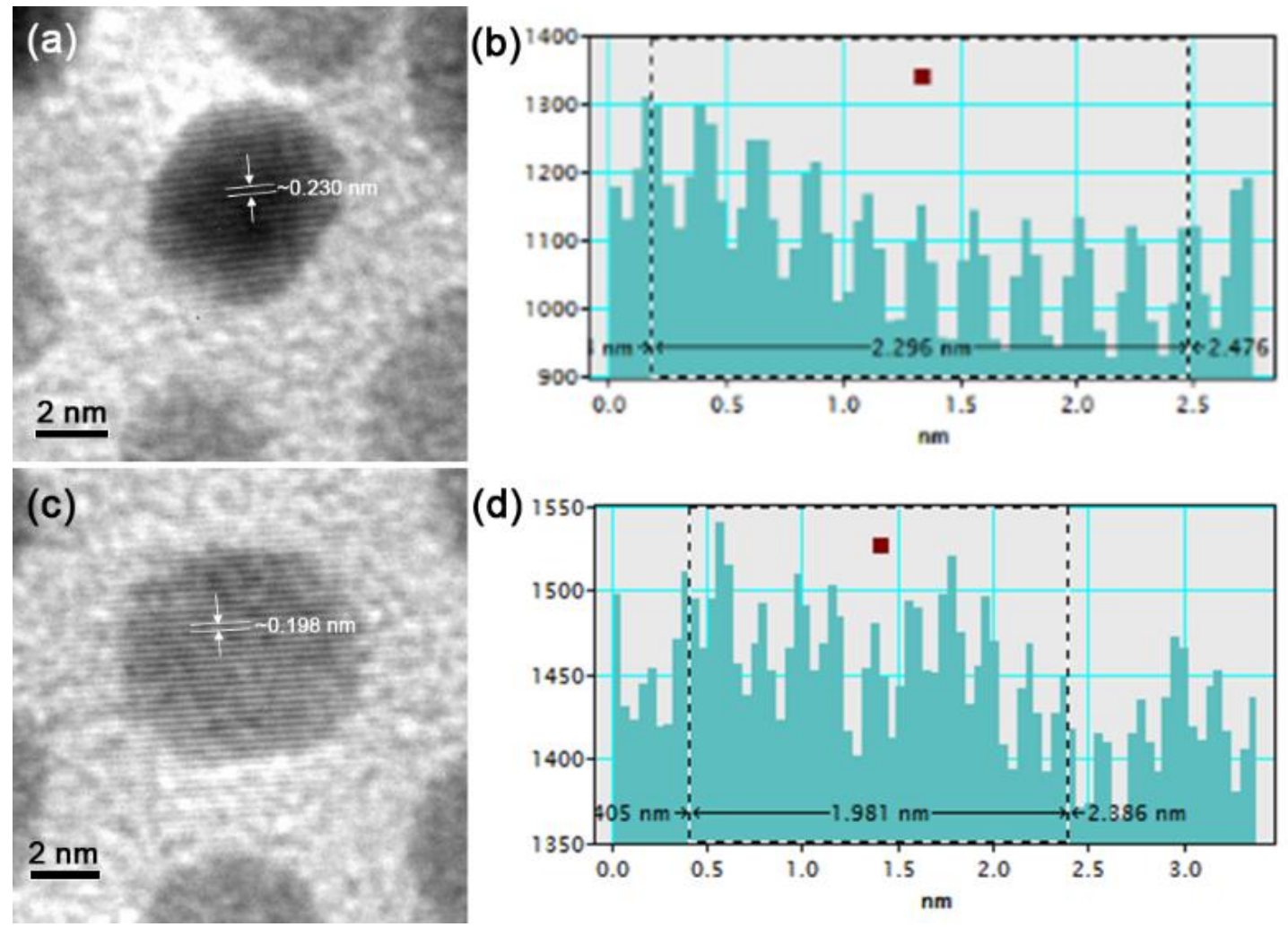

Figure S6. HRTEM images of polyhedron of (a) $\mathrm{Pt}_{9} \mathrm{Fe}_{1}\{111\}$ plane, and (c) $\mathrm{Pt}_{9} \mathrm{Fe}_{1}$ $\{100\}$ plane. The calculated results, obtained though the analysis of selected area with 10 intervals, show the lattice distances of polyhedron of (b) $\mathrm{Pt}_{9} \mathrm{Fe}_{1}\{111\}$ plane, and (d) $\mathrm{Pt}_{9} \mathrm{Fe}_{1}\{100\}$ plane. 

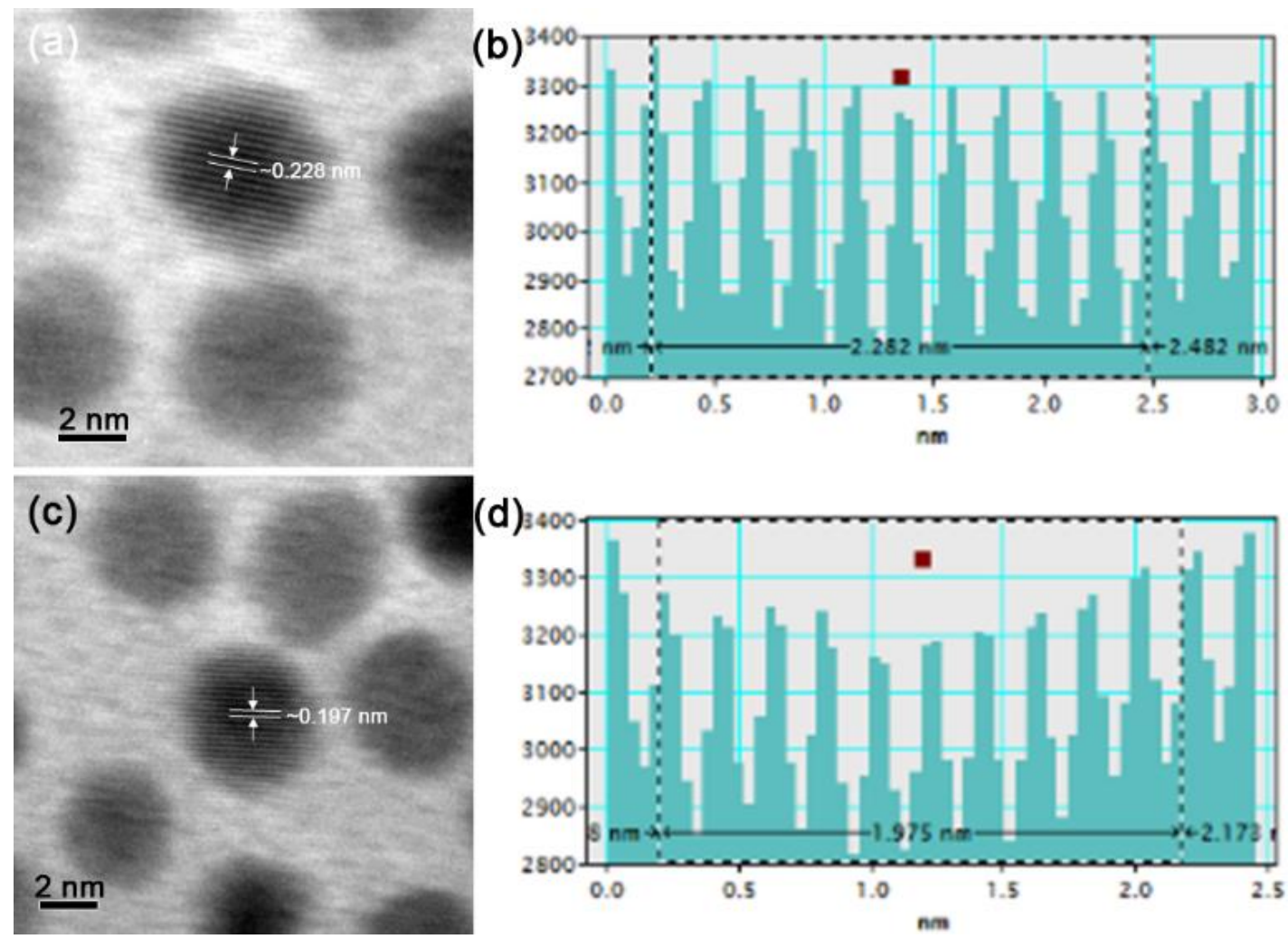

Figure S7. HRTEM images of polyhedron of (a) $\mathrm{Pt}_{7} \mathrm{Fe}_{3}\{111\}$ plane, and (c) $\mathrm{Pt}_{7} \mathrm{Fe}_{3}$ $\{100\}$ plane. The calculated results, obtained though the analysis of selected area with 10 intervals, show the lattice distances of polyhedron of (b) $\mathrm{Pt}_{7} \mathrm{Fe}_{3}\{111\}$ plane, and (d) $\mathrm{Pt}_{7} \mathrm{Fe}_{3}\{100\}$ plane. 

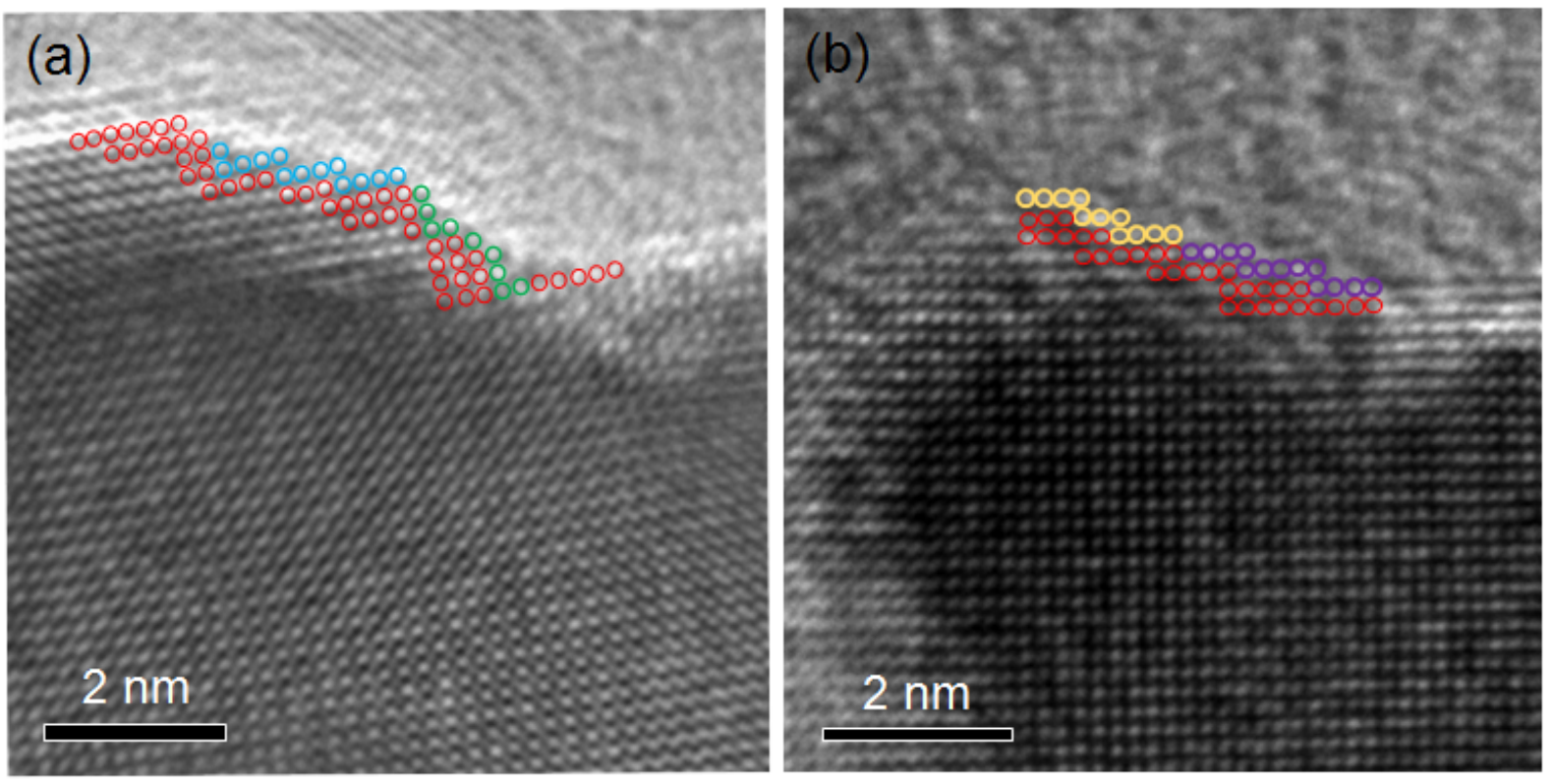

Figure S8. HRTEM images of concave cube of (a) $\mathrm{Pt}_{9} \mathrm{Fe}_{1}$, and (b) $\mathrm{Pt}_{7} \mathrm{Fe}_{3}$. In Figure S5a, the blue cycles indicate the $\{710\}$ facet; the green cycles indicate the $\{420\}$ facets; the red cycles indicate the $\{100\}$ facet. Also, in Figure S5b, the yellow cycles indicate the $\{730\}$ facet; the purple cycles indicate the $\{740\}$ facets; the red cycles indicate the $\{100\}$ facet. 


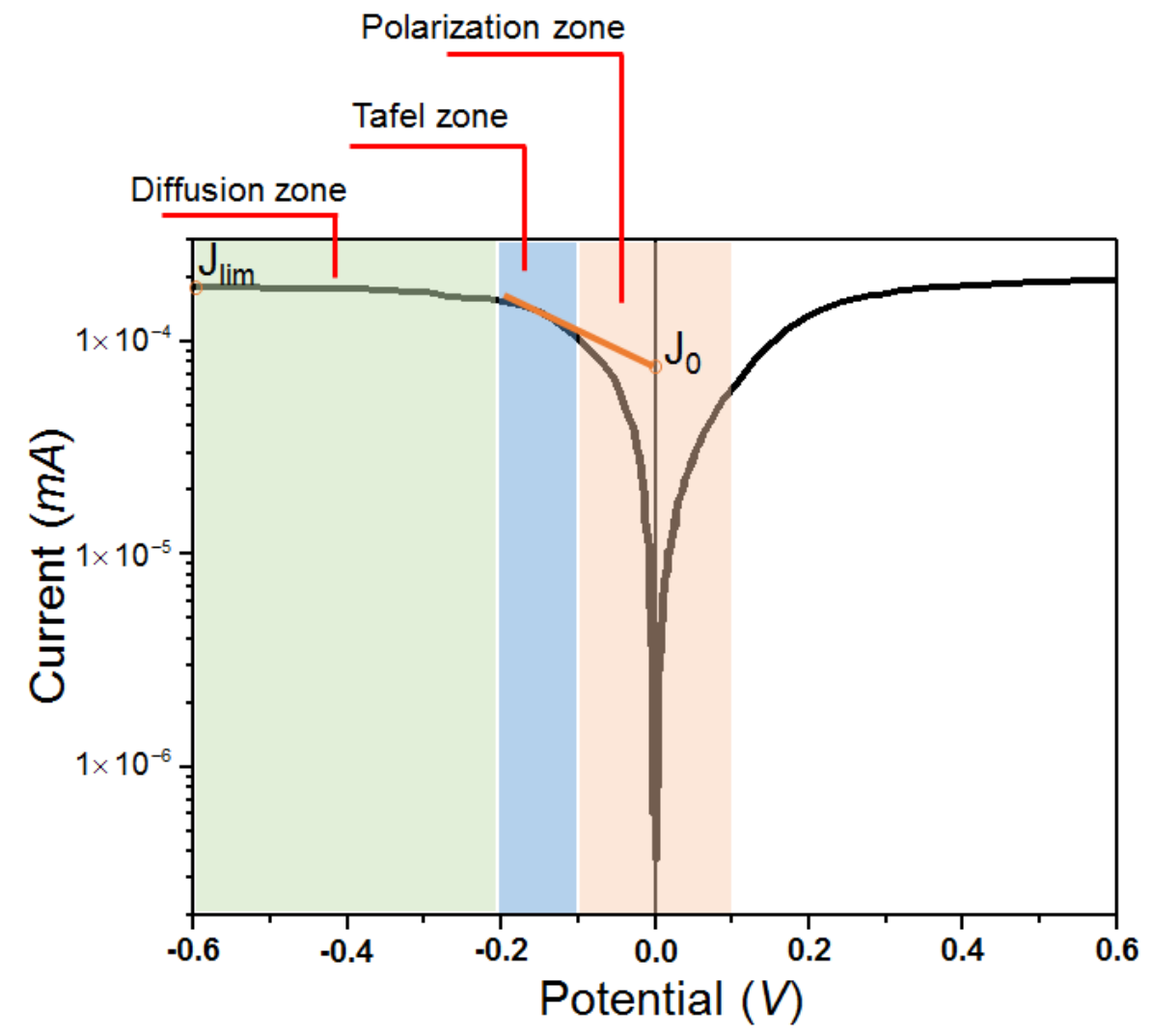

Figure S9. Tafel curves of three-zone diagram including polarization zone, Tafel zone, and diffusion zone. 
(a)

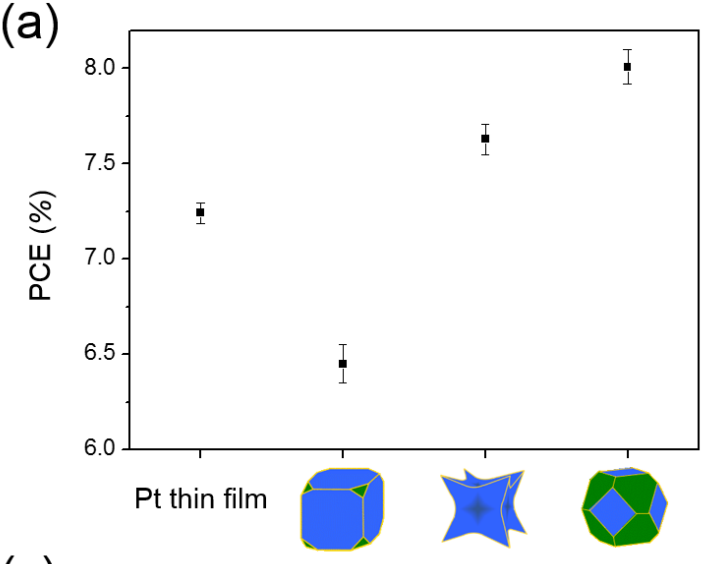

(c)

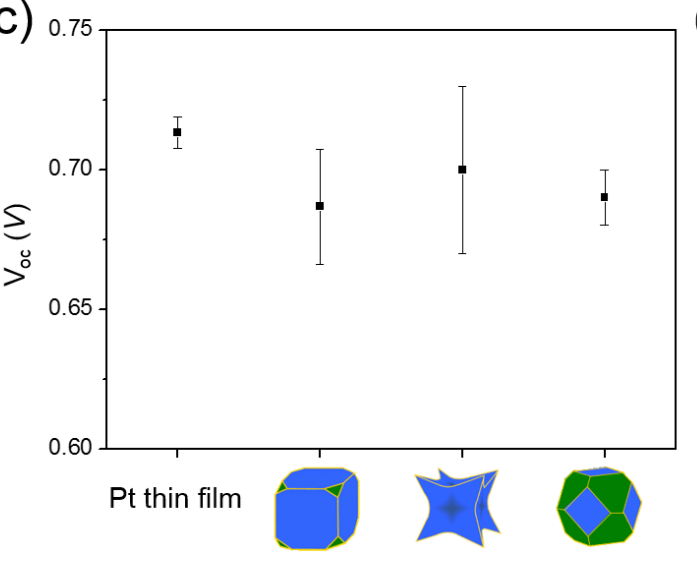

(b)

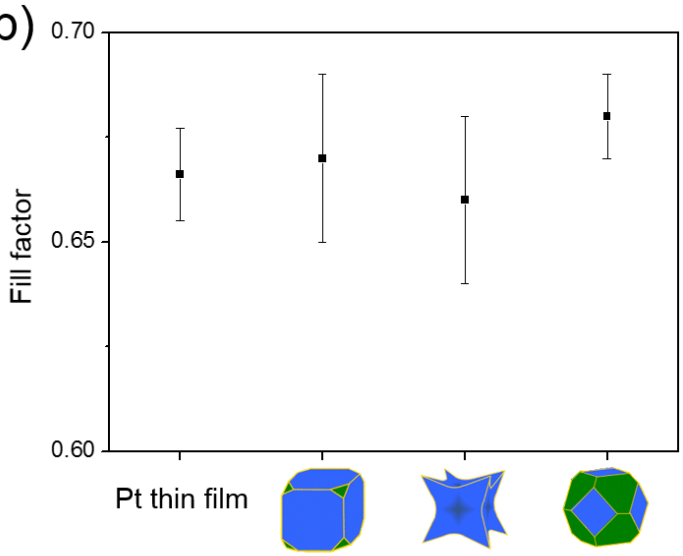

(d)

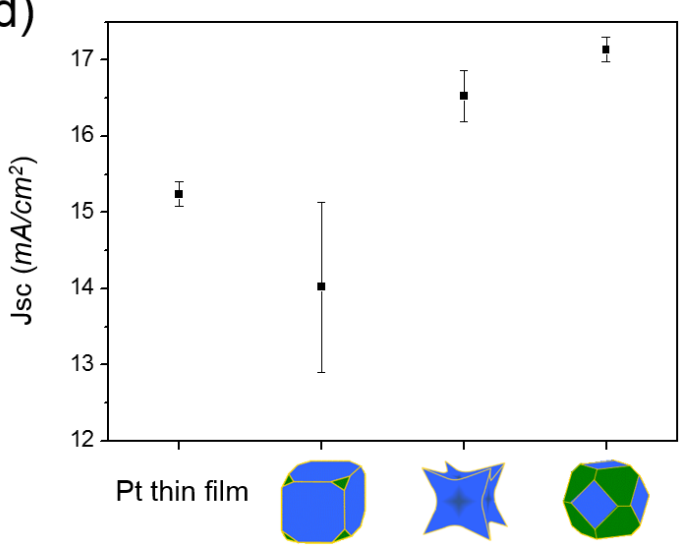

Figure S10. Photovoltaic parameters of DSSC device using the Pt thin film and $\mathrm{Pt}_{9} \mathrm{Fe}_{1}$ nanostructures as CEs. 
(a)

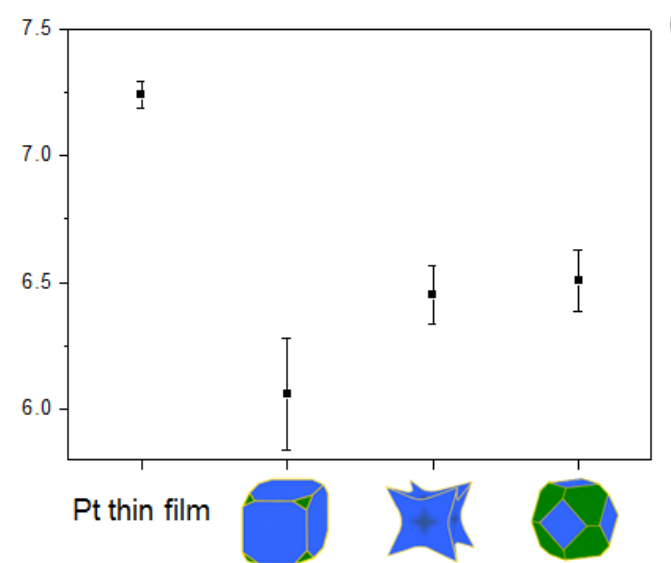

(c)

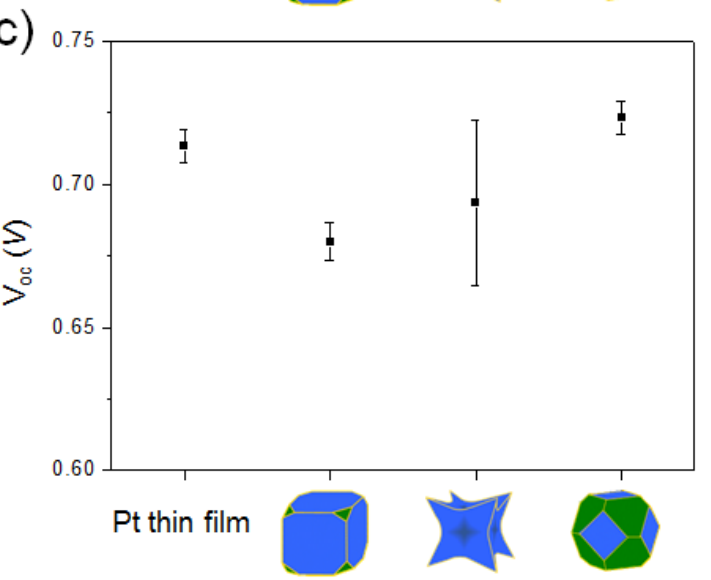

(b)

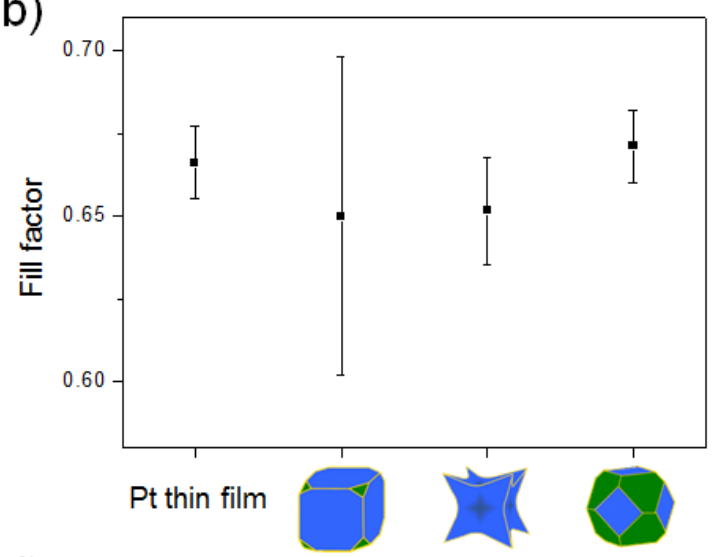

(d)

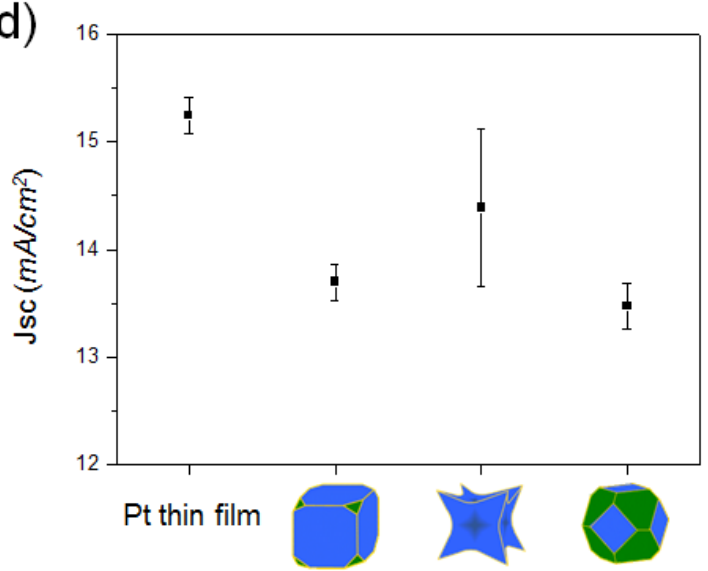

Figure S11. Photovoltaic parameters of DSSC device using the Pt thin film and $\mathrm{Pt}_{7} \mathrm{Fe}_{3}$ nanostructures as CEs. 


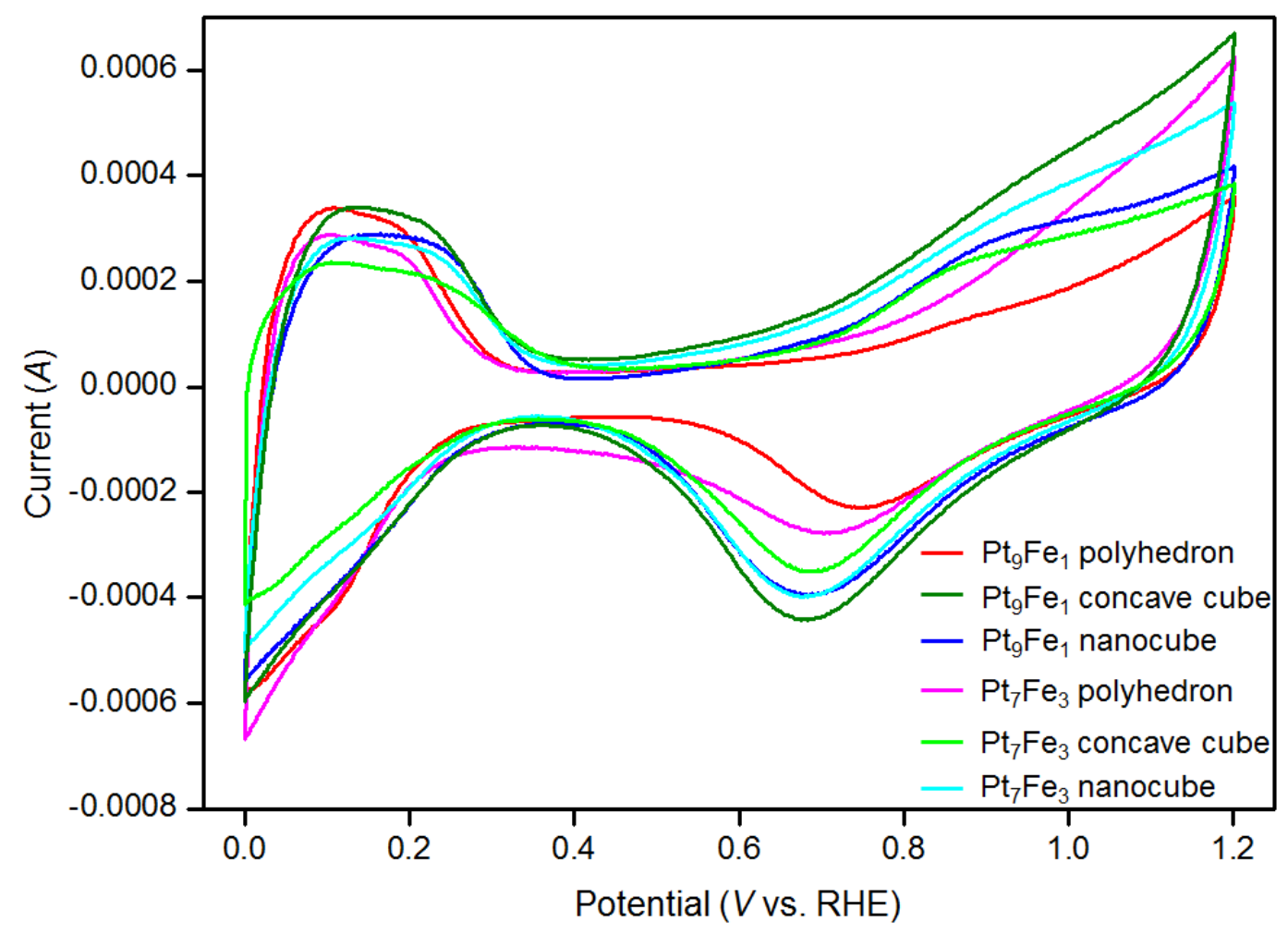

Figure S12. Cyclic voltammograms (CV) of $\mathrm{Pt}_{9} \mathrm{Fe}_{1}$ and $\mathrm{Pt}_{7} \mathrm{Fe}_{3}$ nanostructures obtained in $0.1 \mathrm{M} \mathrm{HClO}_{4}$. 


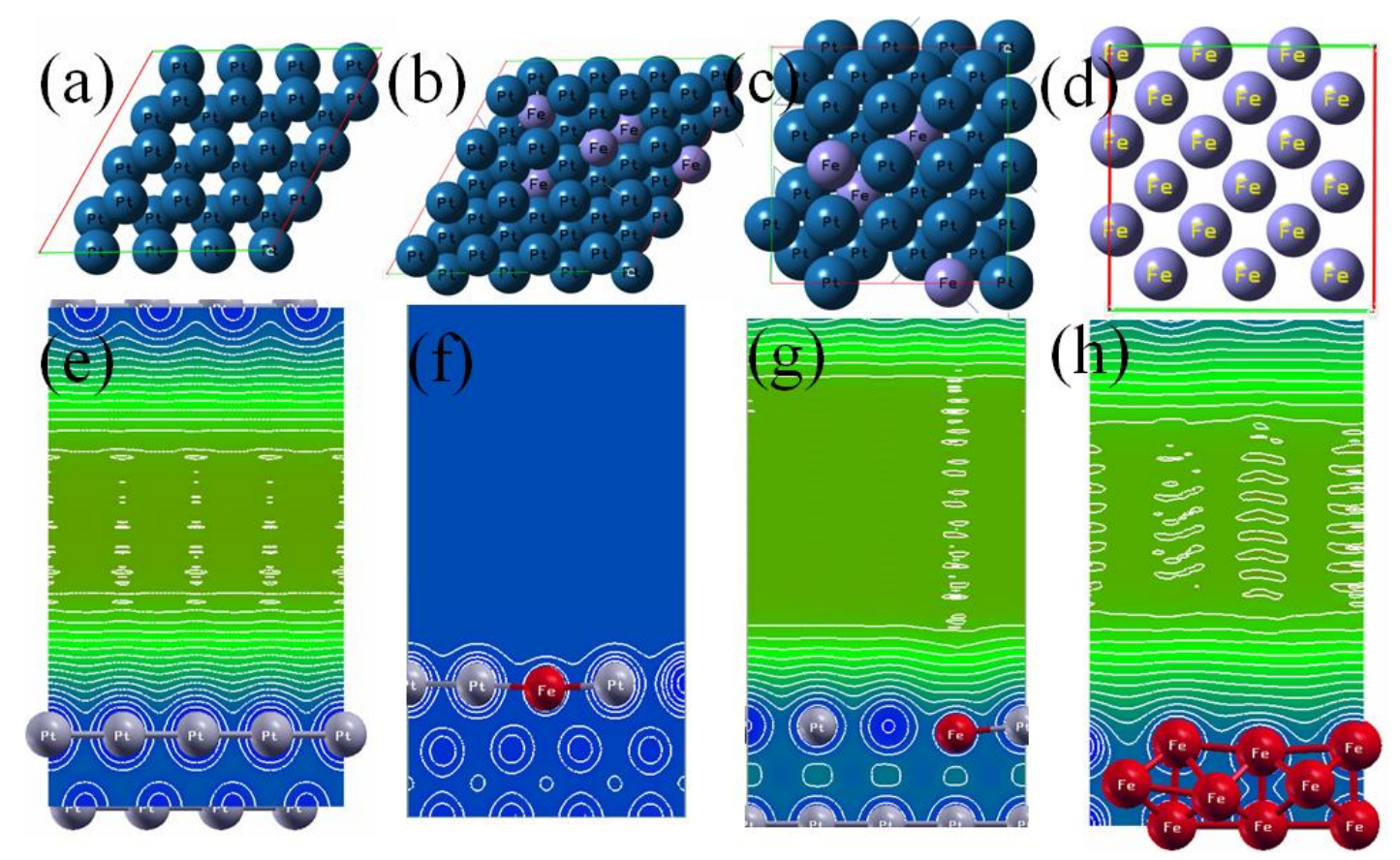

Figure S13. The model of (a) top-view of $\mathrm{Pt}(111)$, and (b) top-view of $\mathrm{Pt}_{9} \mathrm{Fe}_{1}(111)$, and (c) top-view of $\mathrm{Pt}_{9} \mathrm{Fe}_{1}(100)$, and (d) top-view of $\mathrm{Fe}(100)$, and the contour plot of (e) $\mathrm{Pt}(111)$ and (f) $\mathrm{Pt}_{9} \mathrm{Fe}_{1}(111)$ and $(\mathrm{g}) \mathrm{Pt}_{9} \mathrm{Fe}_{1}(100)$, and (h) $\mathrm{Fe}(100)$ slab, respectively. 
Table S1. The experimental parameters of PtFe nanostructures with various alloying composition.

\begin{tabular}{cccccc}
\hline $\mathrm{PtFe}_{2}$ nanostructure & $\mathrm{Pt}(\mathrm{acac})_{2}$ & $\mathrm{Fe}(\mathrm{CO})_{5}$ & $\mathrm{HDD}$ & OLAm & $\mathrm{OA}$ \\
\hline $\mathrm{Pt}_{9} \mathrm{Fe}_{1}$ concave cube & $47 \mathrm{mg}$ & $26 \mu \mathrm{L}$ & $200 \mathrm{mg}$ & $4 \mathrm{~mL}$ & $4 \mathrm{~mL}$ \\
$\mathrm{Pt}_{9} \mathrm{Fe}_{1}$ nanocube & $47 \mathrm{mg}$ & $26 \mu \mathrm{L}$ & $200 \mathrm{mg}$ & $0.3 \mathrm{~mL}$ & $0.6 \mathrm{~mL}$ \\
$\mathrm{Pt}_{7} \mathrm{Fe}_{3}$ polyhedron & $47 \mathrm{mg}$ & $26 \mu \mathrm{L}$ & $200 \mathrm{mg}$ & $0.3 \mathrm{~mL}$ & $2 \mathrm{~mL}$ \\
$\mathrm{Pt}_{7} \mathrm{Fe}_{3}$ concave cube & $47 \mathrm{mg}$ & $60 \mu \mathrm{L}$ & $200 \mathrm{mg}$ & $4 \mathrm{~mL}$ & $4 \mathrm{~mL}$ \\
$\mathrm{Pt}_{7} \mathrm{Fe}_{3}$ nanocube & $47 \mathrm{mg}$ & $60 \mu \mathrm{L}$ & $200 \mathrm{mg}$ & $0.3 \mathrm{~mL}$ & $0.6 \mathrm{~mL}$ \\
$\mathrm{Pt}_{9} \mathrm{Fe}_{1}$ polyhedron & $47 \mathrm{mg}$ & $60 \mu \mathrm{L}$ & $200 \mathrm{mg}$ & $0.3 \mathrm{~mL}$ & $2 \mathrm{~mL}$ \\
\hline
\end{tabular}

All reactants were loaded into 1 -octadecene of $6 \mathrm{~mL}$ and reacted at $240{ }^{\circ} \mathrm{C}$ for $35 \mathrm{~min}$. 
Table S2. Summary of the $\mathrm{H}_{2}$-desorption area, electric charge, active area, and specific area of different counter electrodes. The loading Pt mass for different counter electrodes is as follows: $5.75 \mu \mathrm{g}$ for $\mathrm{Pt}_{9} \mathrm{Fe}_{1}$ polyhedron, $5.71 \mu \mathrm{g}$ for $\mathrm{Pt}_{9} \mathrm{Fe}_{1}$ concave cube, $5.78 \mu \mathrm{g}$ for $\mathrm{Pt}_{9} \mathrm{Fe}_{1}$ nanocube, $5.25 \mu \mathrm{g}$ for $\mathrm{Pt}_{7} \mathrm{Fe}_{3}$ polyhedron, $5.41 \mu \mathrm{g}$ for $\mathrm{Pt}_{7} \mathrm{Fe}_{3}$ concave cube, $5.47 \mu \mathrm{g}$ for $\mathrm{Pt}_{7} \mathrm{Fe}_{3}$ nanocube,..

\begin{tabular}{lcccc}
\hline Counter electrode & $\begin{array}{c}\mathrm{H}_{2} \text {-desorption area } \\
(\mathrm{A} \times \mathrm{V})\end{array}$ & $\begin{array}{c}\text { Electric charge } \\
(\mathrm{C})\end{array}$ & $\begin{array}{c}\text { Active area } \\
\left(\mathrm{cm}^{2}\right)\end{array}$ & $\begin{array}{c}\text { Specific area } \\
\left(\mathrm{cm}^{2} / \mathrm{mg}-\mathrm{Pt}\right)\end{array}$ \\
\hline $\mathrm{Pt}_{9} \mathrm{Fe}_{1}$ polyhdron & $6.11 \times 10^{-5}$ & $6.11 \times 10^{-4}$ & 2.91 & 505.29 \\
\hline $\mathrm{Pt}_{9} \mathrm{Fe}_{1}$ concave cube & $6.18 \times 10^{-5}$ & $6.18 \times 10^{-4}$ & 2.94 & 515.28 \\
\hline $\mathrm{Pt}_{9} \mathrm{Fe}_{1}$ nanocube & $6.05 \times 10^{-5}$ & $6.05 \times 10^{-4}$ & 2.88 & 504.76 \\
\hline $\mathrm{Pt}_{7} \mathrm{Fe}_{3}$ polyhdron & $4.97 \times 10^{-5}$ & $4.97 \times 10^{-4}$ & 2.13 & 405.40 \\
\hline $\mathrm{Pt}_{7} \mathrm{Fe}_{3}$ concave cube & $5.16 \times 10^{-5}$ & $5.16 \times 10^{-4}$ & 2.46 & 454.70 \\
\hline $\mathrm{Pt}_{7} \mathrm{Fe}_{3}$ nanocube & $5.39 \times 10^{-5}$ & $5.39 \times 10^{-4}$ & 2.57 & 469.23 \\
\hline
\end{tabular}


Table S3. Adsorption energies of $\mathrm{I}_{2}$, desorption energies of $\mathrm{I}^{-}$, corresponding absolute energies and optimized geometries calculation of iodine in $\mathrm{Pt}(111), \mathrm{Pt}_{9} \mathrm{Fe}_{1}(111)$ and $\mathrm{Pt}_{9} \mathrm{Fe}_{1}(100)$ surface

\begin{tabular}{ccccc}
\hline Systems & $\begin{array}{c}\mathrm{E}_{\mathrm{ad}} \\
(\mathrm{eV})\end{array}$ & $\begin{array}{c}\mathrm{E}_{\mathrm{de}} \\
(\mathrm{eV})\end{array}$ & $\begin{array}{c}\mathrm{E}_{\mathrm{abs}} \\
(\mathrm{eV})\end{array}$ & $\begin{array}{c}d(\mathrm{Pt}-\mathrm{I}) \\
(\AA)\end{array}$ \\
\hline $\mathrm{Pt}(111)$ & -1.23 & $2.53(\mathrm{a})$ & 1.28 & $2.911(\mathrm{a})$ \\
& & 2.51 & & 2.903 \\
$\mathrm{Pt}_{9} \mathrm{Fe}_{1}(111)$ & -1.10 & 2.21 & 1.11 & 2.734 \\
& & & & 2.723 \\
$\mathrm{Pt}_{9} \mathrm{Fe}_{1}(100)$ & -0.25 & 2.76 & 2.51 & 2.705 \\
& & & & 2.708
\end{tabular}

(a) Jiawei Wan, Guojia Fang, Huajie Yin, Xuefeng Liu, Di Liu, Meiting Zhao, Weijun Ke, Hong Tao, Zhiyong Tang, Adv. Mater. 2014, 26, 8101-8106. 
Table S4. Bader Charger Analysis in $\mathrm{Pt}(111)$ and $\mathrm{Fe}(100)$ and $\mathrm{Pt}_{9} \mathrm{Fe}_{1}(111)$ and $\mathrm{Pt}_{9} \mathrm{Fe}_{1}(100)$ systems.

\begin{tabular}{ccc}
\hline Systems & Charge (e) & Charge difference (e) \\
\hline $\mathrm{Pt}(111)$ & $\mathrm{Pt}: 10.044$ & \\
$\mathrm{Fe}(100)$ & $\mathrm{Fe}: 8.637$ & Pt: $10.044-10.484=-0.442$ \\
\hline $\mathrm{Pt}_{9} \mathrm{Fe}_{1}(111)$ & $\mathrm{Pt}: 10.484$ & Fe: $8.637-7.494=+1.143$ \\
& $\mathrm{Fe}: 7.494$ & $\mathrm{Pt}: 10.044-10.347=-0.303$ \\
\hline $\mathrm{Pt}_{9} \mathrm{Fe}_{1}(100)$ & $\mathrm{Pt}: 10.347$ & $\mathrm{Fe}: 8.637-7.389=+1.248$ \\
& $\mathrm{Fe}: 7.389$ & \\
\hline
\end{tabular}

